\title{
Erratum to: Differential Brain, Cognitive and Motor Profiles Associated with Partial Trisomy. Modeling Down Syndrome in Mice
}

Pierre L. Roubertoux ${ }^{1} \cdot$ Nathalie Baril $^{2} \cdot$ Pierre Cau $^{1,3,4} \cdot$ Christophe Scajola $^{1}$. Adeline Ghata $^{1} \cdot$ Catherine Bartoli $^{1} \cdot$ Patrice Bourgeois $^{1,3} \cdot$ Julie di Christofaro $^{5}$. Sylvie Tordjman ${ }^{6,7} \cdot$ Michèle Carlier $^{8}$

Published online: 23 March 2017

(C) Springer Science+Business Media New York 2017

\section{Erratum to: Behav Genet}

DOI 10.1007/s10519-017-9835-5

The original version of this article unfortunately contained a mistake.

The affiliation of the author Dr. Nathalie Baril was incorrectly displayed as:

Department 3C, Aix Marseille University, CNRS, Marseille, France

instead of
The online version of the original article can be found under doi:10.1007/s10519-017-9835-5.

Pierre L. Roubertoux

Pierre.ROUBERTOUX@univ-amu.fr

1 Aix Marseille University, INSERM, UMR_S 910, GMGF, TIMONE - 27 Boulevard Jean Moulin, 13005 Marseille, France

2 Aix-Marseille Université, CNRS, FR 3512, Marseille, France

3 Department of Medical Genetics, AP-HM, Timone Hospital, Marseille, France

4 Service de Biologie Cellulaire, AP-HM, Hôpital La Timone, 13385 Marseille Cedex 5, France

5 Aix Marseille University, CNRS, EFS, ADES, Marseille, France

6 Paris Descartes University, CNRS, LPP, Paris, France

7 Rennes 1 University, PHUPEA, Rennes, France

8 Aix Marseille University, CNRS, LPC, Marseille, France
Aix-Marseille Université, CNRS, FR 3512, Marseille, France.

The error is corrected with this erratum. 